\title{
Dual Role of Insulin-Like Growth Factor (IGF)-I in American Tegumentary Leishmaniasis
}

\author{
Carolina de 0 Mendes-Aguiar $\mathbb{D}^{1,2}$ Camilla Lopes-Siqueira, ${ }^{1}$ Fabrício Pettito-Assis $\mathbb{D},^{3}$ \\ Márcia Pereira-Oliveira ${ }^{D},{ }^{1}$ Manoel Paes de Oliveira-Neto, ${ }^{4}$ Claude Pirmez ${ }^{1 D},{ }^{1}$ \\ Alda Maria Da-Cruz $\left(\mathbb{D},{ }^{1,5}\right.$ and Hiro Goto $\mathbb{D}^{3}$ \\ ${ }^{1}$ Laboratório Interdisciplinar de Pesquisas Médicas, Instituto Oswaldo Cruz, FIOCRUZ, Rio de Janeiro, Brazil \\ ${ }^{2}$ Instituto de Medicina Tropical do Rio Grande do Norte, Universidade Federal do Rio Grande do Norte, RN, Brazil \\ ${ }^{3}$ Instituto de Medicina Tropical de São Paulo, Faculdade de Medicina, Universidade de São Paulo, São Paulo, Brazil \\ ${ }^{4}$ Instituto Nacional de Infectologia Evandro Chagas, FIOCRUZ, Rio de Janeiro, Brazil \\ ${ }^{5}$ Disciplina de Parasitologia, DMIP, Faculdade de Ciências Médicas, Universidade do Estado do Rio de Janeiro, Brazil
}

Correspondence should be addressed to Hiro Goto; hgoto@usp.br

Received 21 December 2020; Revised 12 February 2021; Accepted 10 March 2021; Published 30 March 2021

Academic Editor: Luiz Felipe Domingues Passero

Copyright (c) 2021 Carolina de O Mendes-Aguiar et al. This is an open access article distributed under the Creative Commons Attribution License, which permits unrestricted use, distribution, and reproduction in any medium, provided the original work is properly cited.

Background. Cytokines and growth factors involved in the tissue inflammatory process influence the outcome of Leishmania infection. Insulin-like growth factor (IGF-I) constitutively present in the skin may participate in the inflammatory process and parasite-host interaction. Previous work has shown that preincubation of Leishmania (Leishmania) amazonensis with recombinant IGF-I induces accelerated lesion development. However, in human cutaneous leishmaniasis (CL) pathogenesis, it is more relevant to the persistent inflammatory process than progressive parasite proliferation. In this context, we aimed to investigate whether IGF-I was present in the CL lesions and if this factor may influence the lesions' development acting on parasite growth and/or on the inflammatory/healing process. Methodology. Fifty-one CL patients' skin lesion samples from endemic area of L. (Viannia) braziliensis infection were submitted to histopathological analysis and searched for Leishmania and IGF-I expression by immunohistochemistry. Results. In human CL lesions, IGF-I was observed preferentially in the late lesion (more than 90 days), and the percentage of positive area for IGF-I was positively correlated with duration of illness $(r=0.42, P<0.05)$. IGF-I was highly expressed in the inflammatory infiltrate of CL lesions from patients evolving with good response to therapy $(2.8 \% \pm 2.1 \%$; median $=2.1 \% ; n=18)$ than poor responders $(1.3 \% \pm 1.1 \%$; median: $1.05 \% ; n=6 ; P<0.05)$. Conclusions. It is the first time that IGF-I was detected in lesions of infectious cutaneous disease, specifically in American tegumentary leishmaniasis. IGF-I was related to chronicity and good response to treatment. We may relate this finding to the efficient anti-inflammatory response and the known action of IGF-I in wound repair. The present data highlight the importance of searching nonspecific factors besides adaptive immune elements in the study of leishmaniasis' pathogenesis.

\section{Introduction}

Leishmaniases are diseases caused by protozoan parasites of the genus Leishmania, endemic in around 88 countries. In Brazil, L. (Viannia) braziliensis is the main species for American tegumentary leishmaniasis (ATL), causing injuries ranging from benign cutaneous to disfiguring mucosal lesions [1]. After promastigote inoculation in the skin, these parasites interact primarily with innate host elements and growth fac- tors present in this site. Once established the infection, the inflammatory infiltrate in the cutaneous leishmaniasis (CL) is characterized as chronic inflammation, with granuloma with or without necrosis, and the presence of macrophages, plasma cells, and lymphocytes [2]. The disease's progression is not directly correlated to progressive parasite growth in the lesion site, with few parasites being detected mainly in chronic cases [3]. Instead, cell-mediated immunity has essential participation in CL pathogenesis. Chronic CL lesions are 
composed of an increased number of activated $\mathrm{CD}^{+} 9^{+} \mathrm{T}[4]$ and regulatory $\mathrm{CD} 4{ }^{+} \mathrm{CD} 25^{+} \mathrm{FOXP} 3^{+} \mathrm{IL}$ - 10 -producing $\mathrm{T}$ cells $[5,6]$, granzyme A CD8 ${ }^{+}$cytotoxic T cells, or even proinflammatory $\mathrm{CD}^{+}{ }^{+}$IFN- $\gamma$-producing $\mathrm{T}$ cells [6]. Further, in a recent transcriptomic study in skin samples of ATL patients, delayed or no cure was correlated to the higher expression of gene sets related to the cytolytic pathway [7]. These findings exemplify the complexity of CL immunopathogenesis.

In the skin, different innate elements and growth factors participate at the inoculation site of the parasite. Insulinlike growth factor (IGF)-I is one of them, and we have been studying its participation in Leishmania-vertebrate host interaction. It is a hormone that acts as an autocrine and/or paracrine element, being essential to maintain body homeostasis. It can be detected in the serum, but it has a widespread distribution in tissues [8]. Many cells, including macrophages, produce IGF-I [8-10]. Different IGF-I serum levels were associated with the pathogenesis of melanomas [11], HPV infection [12], and psoriasis [13]. In cutaneous tissues, IGF-I has a central role in wound healing [14]. In leishmaniasis, previous studies have demonstrated the effect of IGF-I in inducing in vitro proliferation of different species of Leishmania $[15,16]$. In vivo, in mouse model of CL, an increase in lesion size and the number of viable parasites after infection with IGF-I preactivated promastigotes of L. amazonensis was observed [17]. IGF-I induces arginase activation, which in turn activates Leishmania promastigotes [18]. Moreover, infection with IGF-I-preactivated Leishmania leads to an increase in lesion size in mice, due to the expansion of the inflammatory infiltrate and parasite growth, suggesting that IGF-I may contribute to cell migration besides parasite proliferation [17]. However, in ATL patients with mucosal and disseminated forms, IGF-I serum levels were lower than in simple CL and healthy controls [19].

IGF-I can potentially interact with Leishmania parasites in the initial phase of infection. Nevertheless, it is still unknown whether IGF-I has any role in the later stages of infection and whether this growth factor may contribute to leishmaniases' pathogenesis. Therefore, we aimed to investigate whether IGF-I was present in the CL lesions and if this factor may influence the development of the lesion acting on parasite growth and/or on the inflammatory/healing process.

\section{Subjects and Methods}

2.1. Growth Curve of Leishmania spp. in the Presence of IGF-I. L. (V.) braziliensis (MHOM/BR/1975/M2903) promastigotes were maintained at $25^{\circ} \mathrm{C}$, in Schneider's insect medium (Gibco, Thermo Fisher Scientific Inc, MA, USA) supplemented with $10 \%$ heat-inactivated fetal calf serum (Gibco, USA) and $200 \mathrm{IU}$ of penicillin per $\mathrm{mL}$, and $200 \mu \mathrm{g}$ of streptomycin (Sigma Chemical Company, St. Louis, Mo, USA) per $\mathrm{mL}$, and grown until stationary phase before using in Leishmania growth curve analysis. The stationary phase promastigotes were distributed in triplicate into 24 -well plates $\left(5 \times 10^{5}\right.$ parasites/well $)$ in a final volume of $1.0 \mathrm{~mL}$ of Schneider's insect medium (Gibco, USA) supplemented with $2 \%$ FCS and antibiotics, with or without $50 \mathrm{ng} / \mathrm{mL}$ recombi- nant human IGF-I (rIGF-I, R\&D Systems, USA) and maintained during the experimental period.

2.2. Patients. The patient groups were composed of 37 men and 14 women, all of them living in endemic area of $L$. braziliensis transmission. The median age was 35 [18-57] years. The patients were classified according to the duration of illness at the moment of diagnosis as early group when the appearance of the lesions was less than 30 days before; intermediate, in the 30-60-day interval; late, more than 90 days. The patients were also grouped by having a good response (complete epithelization three months after the end of therapy) or poor response (no complete healing of lesions three months after the end of treatment or development of secondary lesions).

The following criteria were used for leishmaniases diagnosis: (i) type of lesion and epidemiological data compatible with ATL; (ii) positive delayed-type hypersensitivity reaction to leishmanial antigens; (iii) detection of serum anti-Leishmania antibodies; and (iv) detection of Leishmania parasites in lesion by microscopic examination of histological sections or by culture in NNN-modified medium. Patients were treated with pentavalent antimony ( $N$-methyl-glucamine, Glucantime). All procedures were approved by the Ethical Committee of the Fundação Oswaldo Cruz, Ministério da Saúde, Rio de Janeiro, Brazil, and informed consent was obtained from each subject.

The skin fragment was obtained for diagnosis before treatment, and it was taken from the border of the cutaneous lesion. The fragment was divided into three parts: one was fixed for histopathology analysis, the second one was cryopreserved for immunohistochemistry analysis, and the last one was used for Leishmania DNA detection by PCR. Fragments of skin lesions were not taken sequentially after treatment for ethical reasons.

2.3. Immunohistochemistry. Skin fragments were frozen in OCT resin (Tissue Tek; Sakura Finetek, Torrance, CA, U.S.A.), were cut into 3-4 $\mu \mathrm{m}$ thick sections and mounted on microscope slides (silanized slides; DakoCytomation, Carpinteria, CA, USA). To detect IGF-I expression, the slides were fixed in acetone:methanol:formalin $(19: 19: 2)$ and rehydrated in Tris-Saline Buffer (TBS) pH 7.6. The procedure was performed according to the Envision Double Staining kit (Dako, USA) manual. Briefly, endogenous enzymes were blocked with Endogenous Enzyme Block regent, and then, the slides were incubated with the first primary polyclonal rabbit anti-human IGF-I antibody (1:200; GroPep Limited-Adelaide, Australia) followed by incubation with the mouse and rabbit antibody conjugated to Polymer/HRP reagent. This first antibody was developed by $\mathrm{DAB}+$ Chromogen. For the second part of immunostaining, beginning with the blocking step, the Doublestain Block reagent was used. The anti-Leishmania mouse serum was used as a second antibody and was added diluted $1: 8000$. This anti-Leishmania serum was obtained at two months of infection from $\mathrm{BALB} / \mathrm{c}$ mice infected in the footpad with $10^{6}$ stationary phase L. amazonensis promastigotes [20]. Then, mouse and rabbit antibodies conjugated to Polymer/AP reagent were 
used, and permanent Red Chromogen developed the second reaction. Between the steps, the slides were washed with TBS $\mathrm{pH} 7.6$, and the antibodies were diluted in BSA 2\%-Tris-HCL $0.01 \mathrm{M} \mathrm{pH} 7.6$ and incubated for 30 minutes, at room temperature each. At the end of the procedure, the slides were counterstained with Meyer's hematoxylin (Merck, Germany). For control purposes, the specific antibodies were omitted. The slides were examined under a light microscope (Nikon, Nikon, Eclipse E600, Japan).

2.3.1. Immunohistochemistry Analysis. Two independent observers analyzed the slides. All fragment area was analyzed, and the IGF-I and anti-Leishmania staining were classified in only IGF-I detection, IGF-I and Leishmania antigen coexpression, or only Leishmania antigen detection. Twenty-five cases in which IGF-I was present were photographed. Five representative areas were photographed using Cool SnapPro Color (Media Cybernetics Inc, USA), and the photos were analyzed by Image-Pro Plus ${ }^{\circledast}$ Software (Media Cybernetics Inc, USA). The percentage of the positive area was measured for IGF-I and Leishmania sp. antigen staining.

2.4. Statistical Analysis. Mann-Whitney test, Kruskal-Wallis test, and Spearman correlation were utilized (GraphPad Software; San Diego, CA, USA) using the software GraphPad Prism version 6 . Results were considered statistically different when the $P$ value was $\leq 0.05$.

\section{Results}

3.1. Leishmania (Viannia) braziliensis in vitro Growth upon IGF-I Stimulus. In a previous study, the effect of IGF-I on parasite growth in vitro $L$. braziliensis-infected human monocytic cell line THP1 was inconclusive [19]; thus, we first investigated whether IGF-I can influence the L. braziliensis growth in vitro. The addition of rIGF-I in promastigote cultures in physiological concentration increased the L. braziliensis proliferation compared with controls without IGF-I (Figure 1).

\subsection{IGF-I Was Present in Cutaneous Leishmaniases Lesions.} Next, we investigated the presence of IGF-I in fifty-one CL lesions by immunohistochemistry. IGF-I was present in $70.5 \%$ of cases $(n=36)$. Histologically, IGF-I was spread in the dermis and epidermal basal lamina (Figures 2(b) and $2(\mathrm{c})$ ). In normal skin (control; $n=3$ ), IGF-I was seen only in the basal lamina (Figure 2(a)).

Concomitantly with IGF-I immunostaining, we carried out the Leishmania antigen detection. Leishmania antigens were detected in $84.3 \%$ of cases $(n=43)$. Isolated Leishmania antigen detection was found in $29.5 \%$ of cases $(n=15)$. Leishmania antigens were observed in the extracellular matrix and cell foci in the dermis (Figure 2(d)). Leishmania antigen detection decreased with chronicity: $92.8 \%$ of patients $(13 / 14)$ in the early group; in $89.4 \%$ of patients $(17 / 19)$ in the intermediate group; and in $72.2 \%$ of patients (13/18) in the late lesion group (Table 1). IGF-I and Leishmania antigens were observed in the dermis of the same lesion area in 28 cases. However, IGF-I and Leishmania antigens' colocalization was not observed.

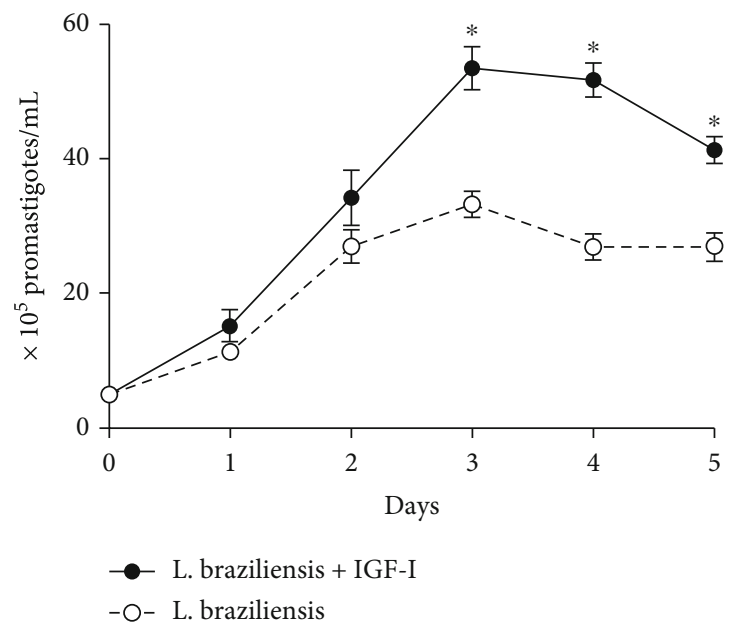

FIGURE 1: Effect of insulin-like growth factor (IGF)-I on Leishmania (Viannia) braziliensis promastigote proliferation. In vitro $L$. braziliensis growth curve in the presence (black circle) or absence (white circle) of $50 \mathrm{ng} / \mathrm{mL}$ rIGF-I. Live promastigotes were counted every day for five days. The points represent the mean of the three experiments, and the bars represent standard deviation. ${ }^{*} P<0.05$

3.3. The Duration of Illness and Treatment Response in relation to the Area of Expression of IGF-I. To evaluate the relationship of the presence of IGF-I with clinical parameters, 36 IGF-I positive cases were grouped according to the duration of illness or treatment response.

IGF-I seems to have a relationship to the chronicity of the lesions since the number of cases expressing IGF-I increases with the disease's progression. The IGF-I was detected in $64.2 \%$ of patients $(9 / 14)$ in the early lesion group, in $68.4 \%$ of patients (13/19) in the intermediate group, and in $77.7 \%$ of patients (14/18) in the late lesion group (Table 1). Moreover, percentage of positive area of IGF-I was also correlated with duration of illness (early lesion group: mean $=1.52 \% \pm 1.12$, median $=1.47 \%, n=7$; intermediate group: mean $=2.32 \% \pm$ 2.47 , median $=1.5, n=10$; late lesion group: mean $=3.18 \%$ \pm 1.9 , median $=3.12, n=8 ; r=0.42, P=0.023$ ) (Table 2).

To assess the relationship of IGF-I expression with treatment response $(n=24)$, we grouped patients in those having a good response (complete epithelization three months after the end of therapy) or having a poor response (no complete healing of lesions three months after the end of treatment or development of secondary lesions). IGF-I was detected in $75 \%$ of patients with good therapeutic response. Those with good response showed higher percentage of positive areas for IGF-I (good responder: mean $=2.8 \% \pm 2.1$, median $=2.1 \%, n=18$ ) when compared with poor response (poor responder: mean: $1.3 \% \pm 1.1$, median $=1.05 \%, n=6$; $P=0.03$ ) (Table 2).

\section{Discussion}

In this work, we initially showed the enhanced proliferation of L. braziliensis promastigotes in the presence of IGF-I. Further, IGF-I was detected in human CL lesions, and for the first time, it was shown in lesions of an infectious cutaneous 


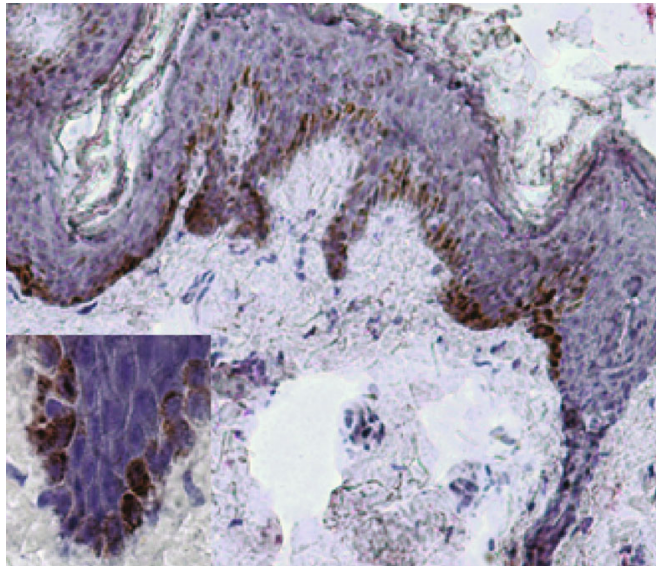

(a)

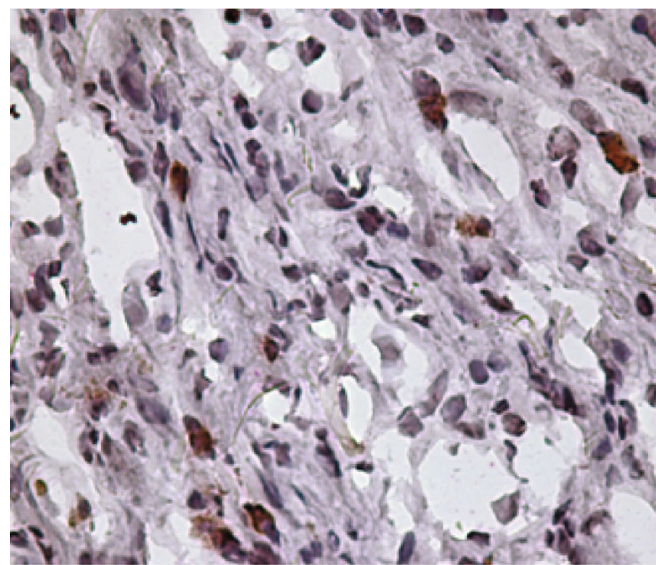

(c)

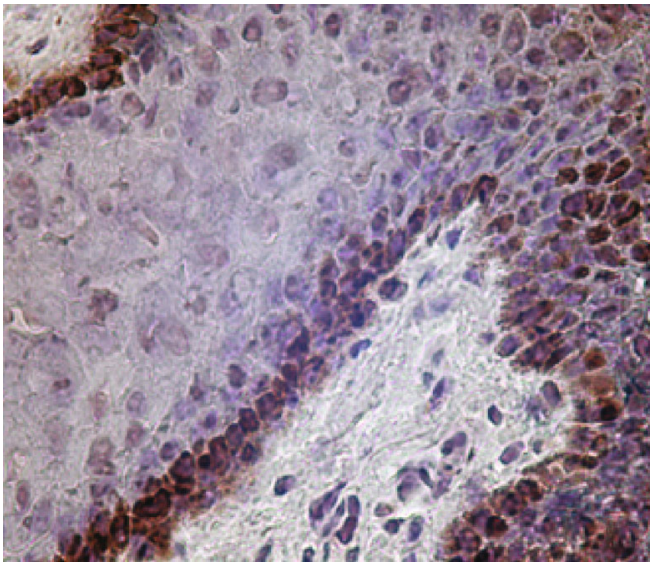

(b)

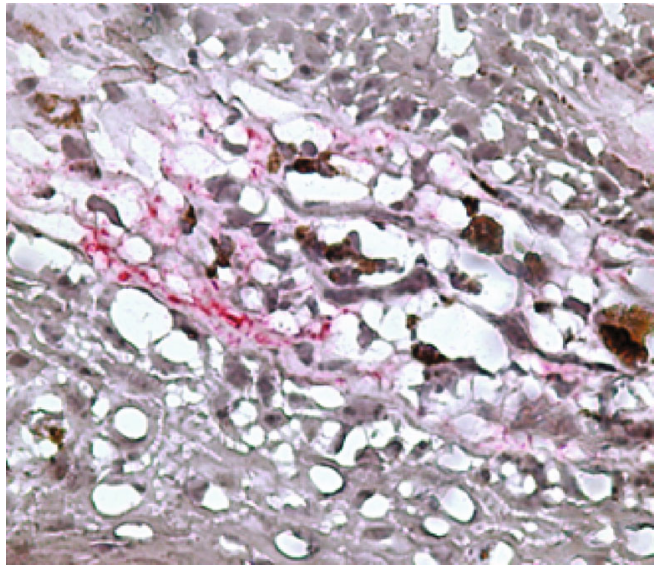

(d)

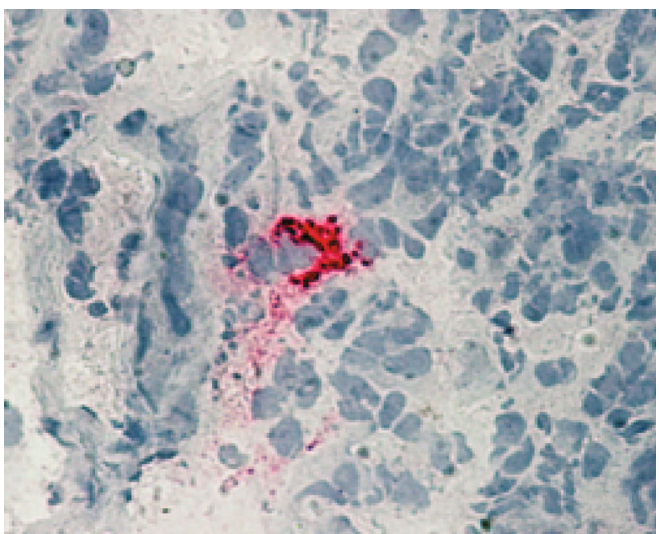

(e)

FIGURE 2: In situ detection of insulin-like growth factor (IGF)-I and Leishmania antigens in the inflammatory infiltrate of cutaneous leishmaniases lesions caused by L. (Viannia) braziliensis. IGF-I (brown) and Leishmania antigens (red) are expressed in normal skin (a) and cutaneous leishmaniases lesions (b-e). IGF-I is present in the basal layer in normal skin (a) and in CL lesions (b). In CL lesions, IGF-I is present in the dermis (c). IGF-I was observed close to Leishmania antigens in the dermis (d). Leishmania antigens were observed in the dermis (e). Representative photos of immunohistochemical analysis. Original magnification $\times 400$.

disease. A limitation of this study was the unfeasibility to obtain sequential material from individual patients due to ethical issues, and further, we had patients who had a different time of development of the overt disease at the moment of diagnosis. However, we were able to analyze the histopathological alterations relating them to the duration of disease and also to response to treatment. Then, the percentage of patients presenting IGF-I detectable in the lesion was higher in chronic lesions. It was also higher among good responders for treatment.

IGF-I interacts with several Leishmania promastigote species inducing proliferation [15-17]. This effect did not 
TABLE 1: Insulin-like growth factor (IGF)-I or Leishmania antigen detection in lesions of cutaneous leishmaniases patients according to the duration of illness.

\begin{tabular}{lccr}
\hline & \% of positive cases & Duration of illness & $\%$ detection $\left(n / n_{\text {total }}\right)$ \\
\hline Leishmanial antigens & & Early & $92.8 \%(13 / 14)$ \\
& $84.3 \%(n=43)$ & Intermediate & $89.4 \%(17 / 19)$ \\
\hline & & Late & $72.2 \%(13 / 18)$ \\
IGF-I & & Early & $64.2 \%(9 / 14)$ \\
& $70.5 \%(n=36)$ & Intermediate & $68.4 \%(13 / 19)$ \\
\hline
\end{tabular}

Duration of illness: early = lesions with less than 30 days; intermediate = lesions with 30-60 days; late = lesions with more than 90 days.

TABLE 2: Area of expression of insulin-like growth factor (IGF)-I in the lesions of cutaneous leishmaniasis patients according to the duration of illness and response to treatment.

\begin{tabular}{|c|c|c|c|c|}
\hline & \multirow{2}{*}{ Groups } & \multicolumn{2}{|c|}{ IGF-I-area \% } & \multirow{2}{*}{$P$} \\
\hline & & Median & Mean \pm SD & \\
\hline \multirow{3}{*}{ Duration of illness } & Early lesion $(n=7)$ & 1.47 & $1.52 \pm 1.12$ & \multirow{3}{*}{$\begin{array}{c}{ }^{* *} r=0.42 \\
0.023\end{array}$} \\
\hline & Intermediate $(n=10)$ & 1.50 & $2.32 \pm 2.47$ & \\
\hline & Late lesion $(n=8)$ & 3.12 & $3.18 \pm 1.90$ & \\
\hline \multirow{2}{*}{ Treatment response } & Good responder $(n=18)$ & 2.10 & $2.80 \pm 2.10$ & \multirow{2}{*}{${ }^{\#} 0.03$} \\
\hline & Poor response $(n=6)$ & 1.05 & $1.30 \pm 1.10$ & \\
\hline
\end{tabular}

${ }^{* *}$ Spearman correlation, ${ }^{*}$ Mann-Whitney test.

occur with IGF-II polypeptide, which shares $60 \%$ of similarity with IGF-I [15]. Here, we showed L. braziliensis promastigote growth in culture in the presence of a physiological concentration of IGF-I as previously observed for other Leishmania species. Thus, we would suggest that IGF-I might act at the beginning of infection interacting with promastigotes at the moment of Leishmania inoculation in the host's skin. With other species, amastigotes were also shown to proliferate in the presence of physiological concentrations of IGF-I [16] but inconclusive with L. braziliensis [19]. IGF-I binds to a receptor expressed on the Leishmania parasite surface, causing phosphorylation of certain proteins and increase the replicative rate of both parasite forms (promastigote and amastigote) in culture [16].

In this work, we analyzed the presence of IGF-I in CL lesions. It is the first data on the presence and influence of in situ IGF-I in human cutaneous infectious disease. In CL lesions, IGF-I was found spread throughout the dermis, basal lamina, and epidermis differing from normal skin where it was seen only in basal lamina. IGF-I's capacity to continuously stimulate keratinocyte growth [9] may contribute to altering the tissue architecture and, at first glance, maybe connected to acanthosis often present in CL lesions [21]. In psoriasis, a cutaneous inflammatory disease, IGF-I contributes to lesion severity stimulating continuous keratinocyte growth [22]. Thus, we hypothesize that the presence of IGF-I in CL lesions may influence the disease outcome.

IGF-I presence in CL lesions with different distribution and a constitutive presence of IGF-I in normal skin leads us to ask whether this factor could act in different phases of Leishmania infection. To address this question, we have performed a double immunostain to IGF-I and Leishmania antigens by immunohistochemistry. Leishmania antigens were detected mainly in recent lesions confirming previous observations [23]. In the mouse model, the infection with IGF-I preactivated $L$. amazonensis promastigotes induced larger lesions than non-pre-activated parasites [17]. The aggravation of mice CL lesions was due to an increase in the number of parasites along with intense cell migration. However, in the present study, we could not observe colocalization of IGF-I and Leishmania antigens in CL lesions suggesting another role for IGF-I in human ATL other than on parasite proliferation. In in vitro study, it was shown intrinsic IGF-I surrounding L. major within infected macrophages [24], but it could be due to the differences among Leishmania species and in this particular experimental conditions, very far from the lesion pathogenic process in the skin.

Here, we also correlated IGF-I expression with lesion chronicity and treatment response. Our results showed that area of IGF-I expression was positively correlated with duration of illness (chronicity). Many immune factors can contribute to chronic CL lesions in humans [6], where the lesion development is related instead to hypersensitivity than to susceptibility to parasite growth $[25,26]$. In this context, increasing concentration of IGF-I in chronic lesions may suggest IGF-I's known participation in anti-inflammatory response $[27,28]$, counteracting persistent, and intense inflammation.

Analyzing the IGF-I area concerning treatment response, we observed higher IGF-I expression areas in patients with good response to the treatment. We may relate this finding to the efficient anti-inflammatory response and the known 
action of IGF-I in wound repair in the reepithelialization [29]. Retarded wound healing in mice diabetic lesions was associated with a delay in IGF-I expression [30]. In humans, a lack of IGF-I expression in diabetes mellitus ulcer contributes to a retarded wound repair [31]. Besides, IGF-I treatment helped to restore the normal expression of both matrix metalloproteinase (MMP)-9 and tissue inhibitors of metalloproteinase (TIMP)-1 in diabetic rats with skin ulcers [32], indicating an important role of IGF-I in healing. Furthermore, in CL patients, MMP-2 was associated with a satisfactory response to antimonial therapy, in conjunction with moderate amounts of IFN- $\gamma$, IL-10, and TGF- $\beta$ [33]. It is conceivable to argue that IGF-I present in CL lesions can act in this net of soluble factors comprising cytokines and hormones involved in the homeostatic process, thus influencing the healing process.

In the pathogenesis of lesion in cutaneous leishmaniasis, inflammatory cytokines [34] and cytotoxic mechanisms [7] are involved that are modulated during development to cure. In this context, the interplay between interferon-gamma (IFN- $\gamma$ ) and IGF-I may determine the outcome of the disease. IFN- $\gamma$ decreases IGF-I expression through inhibition of transcription of IGF-I mRNA [35], and IFN- $\gamma$ is a cytokine that is important for parasite control but implicated in the lesion pathogenesis. In patients with CL in Brazil, expression of IFN- $\gamma$ tends to decrease during the time, mainly in subjects with good response to treatment [36]. Thus, we may speculate that it may result in higher expression of IGF-I in patients with chronic evolution and a good response to treatment.

Our results suggest that IGF-I can play a dual role in CL lesions. IGF-I was detected more in chronic ulcers where may act as an anti-inflammatory factor and in lesions of good responders to treatment where the contribution of this hormone on wound healing would prevail. IGF-I's dual role in CL lesions could be explained by the complex pathogenesis of CL lesions, whereas in the same lesion, we can find areas of an intense inflammatory response and wound repair [34]. It is also observed regions with synthesis besides degradation of extracellular matrix granuloma, compatible with transient or reversible histopathological features occurring in CL lesions [37]. The interplay between IGF-I and other immune-inflammatory elements present in CL lesions may influence the disease outcome.

In the study of leishmaniases' immunity and pathogenesis, most approaches focus on the adaptive immune response that is undoubtedly relevant. However, the present data highlight the importance of searching nonspecific factors such as growth factors besides adaptive immune elements in the study of leishmaniasis' pathogenesis.

\section{Data Availability}

The data used to support the findings of this study are included within the article.

\section{Conflicts of Interest}

There is no conflict of interest.

\section{Acknowledgments}

This work was supported by IOC/FIOCRUZ internal funds, FAPERJ (E-26/102.457/2010), FAPESP (2005/52271-1 and 2018/14398-0), and Laboratório de Investigação Médica (LIM-38), HC-FMUSP. COM-A was a postdoctoral fellow sponsored by FAPERJ/CAPES, Brazil. AMD-C is a $\mathrm{CNPq}$ and FAPERJ (CNE) researcher fellow. $\mathrm{HG}$ is $\mathrm{CNPq}$ research fellow.

\section{References}

[1] M. P. Oliveira-Neto, M. S. Mattos, M. A. Perez et al., "American tegumentary leishmaniasis (ATL) in Rio de Janeiro State, Brazil: main clinical and epidemiologic characteristics," International Journal of Dermatology, vol. 39, no. 7, pp. 506-514, 2000.

[2] A. V. Magalhães, M. A. Moraes, A. N. Raick et al., "Histopathology of cutaneous leishmaniasis by Leishmania braziliensis braziliensis. 1. Histopathological patterns and study of the course of the lesions," Revista do Instituto de Medicina Tropical de São Paulo, vol. 28, no. 4, pp. 253-262, 1986.

[3] M. N. Sotto, E. H. Yamashiro-Kanashiro, V. L. da Matta, and T. de Brito, "Cutaneous leishmaniasis of the New World: diagnostic immunopathology and antigen pathways in skin and mucosa," Acta Tropica, vol. 46, no. 2, pp. 121-130, 1989.

[4] N. L. Diaz, O. Zerpa, L. V. Ponce, J. Convit, A. J. Rondon, and F. J. Tapia, "Intermediate or chronic cutaneous leishmaniasis: leukocyte immunophenotypes and cytokine characterisation of the lesion," Experimental Dermatology, vol. 11, no. 1, pp. 34-41, 2002.

[5] E. Bourreau, C. Ronet, E. Darsissac et al., "In leishmaniasis due to Leishmania guyanensis infection, distinct intralesional interleukin-10 and Foxp3 mRNA expression are associated with unresponsiveness to treatment," The Journal of Infectious Diseases, vol. 199, no. 4, pp. 576-579, 2009.

[6] D. R. Faria, P. E. Souza, F. V. Duraes et al., "Recruitment of $\mathrm{CD}^{+} \mathrm{T}$ cells expressing granzyme $\mathrm{A}$ is associated with lesion progression in human cutaneous leishmaniasis," Parasite Immunology, vol. 31, no. 8, pp. 432-439, 2009.

[7] C. F. Amorim, F. O. Novais, B. T. Nguyen et al., "Variable gene expression and parasite load predict treatment outcome in cutaneous leishmaniasis," Science Translational Medicine, vol. 11, no. 519, 2019.

[8] R. E. Humbel, "Insulin-like growth factors I and II," European Journal of Biochemistry, vol. 190, no. 3, pp. 445-462, 1990.

[9] S. R. Edmondson, S. P. Thumiger, G. A. Werther, and C. J. Wraight, "Epidermal homeostasis: the role of the growth hormone and insulin-like growth factor systems," Endocrine Reviews, vol. 24, no. 6, pp. 737-764, 2003.

[10] J. I. Jones and D. R. Clemmons, "Insulin-like growth factors and their binding proteins: biological actions," Endocrine Reviews, vol. 16, no. 1, pp. 3-34, 1995.

[11] S. L. Park, V. W. Setiawan, P. A. Kanetsky et al., "Serum insulin-like growth factor-I and insulin-like growth factor binding protein-3 levels with risk of malignant melanoma," Cancer Causes \& Control, vol. 22, no. 9, pp. 1267-1275, 2011.

[12] T. G. Harris, R. D. Burk, H. Yu et al., "Insulin-like growth factor axis and oncogenic human papillomavirus natural history," Cancer Epidemiology Biomarkers \& Prevention, vol. 17, no. 1, pp. 245-248, 2008. 
[13] M. El-Komy, I. Amin, A. Zidan, D. Kadry, O. A. Zeid, and O. Shaker, "Insulin-like growth factor-1 in psoriatic plaques treated with PUVA and methotrexate," Journal of the European Academy of Dermatology and Venereology, vol. 25, no. 11, pp. 1288-1294, 2011.

[14] A. Toulon, L. Breton, K. R. Taylor et al., "A role for human skin-resident T cells in wound healing," Journal of Experimental Medicine, vol. 206, no. 4, pp. 743-750, 2009.

[15] C. M. Gomes, H. Goto, C. E. Corbett, and M. Gidlund, "Insulin-like growth factor-1 is a growth promoting factor for Leishmania promastigotes," Acta Tropica, vol. 64, no. 3-4, pp. 225228, 1997.

[16] H. Goto, C. M. Gomes, C. E. Corbett, H. P. Monteiro, and M. Gidlund, "Insulin-like growth factor I is a growthpromoting factor for Leishmania promastigotes and amastigotes," Proceedings of the National Academy of Sciences of the United States of America, vol. 95, no. 22, pp. 13211-13216, 1998.

[17] C. M. Gomes and H. Goto, "Insulin-like growth factor (IGF)-I affects parasite growth and host cell migration in experimental cutaneous leishmaniasis," International Journal of Experimental Pathology, vol. 81, no. 4, pp. 249-255, 2000.

[18] C. M. Vendrame, M. D. Carvalho, F. J. Rios, E. R. Manuli, F. Petitto-Assis, and H. Goto, "Effect of insulin-like growth factor-I on Leishmania amazonensis promastigote arginase activation and reciprocal inhibition of NOS2 pathway in macrophage in vitro," Scandinavian Journal of Immunology, vol. 66, no. 2-3, pp. 287-296, 2007.

[19] L. D. de Souza, C. M. Vendrame, A. R. de Jesus et al., "Insulinlike growth factor-I serum levels and their biological effects on Leishmania isolates from different clinical forms of American tegumentary leishmaniasis," Parasites \& Vectors, vol. 9, no. 1, p. 335, 2016.

[20] F. A. Costa, H. Goto, L. C. Saldanha et al., "Histopathologic patterns of nephropathy in naturally acquired canine visceral leishmaniasis," Veterinary Pathology, vol. 40, no. 6, pp. 677684, 2003.

[21] K. González, R. Diaz, A. F. Ferreira et al., "Histopathological characteristics of cutaneous lesions caused by Leishmania Viannia panamensis in Panama," Revista do Instituto de Medicina Tropical de São Paulo, vol. 60, article e8, 2018.

[22] H. Miura, S. Sano, M. Higashiyama, K. Yoshikawa, and S. Itami, "Involvement of insulin-like growth factor-I in psoriasis as a paracrine growth factor: dermal fibroblasts play a regulatory role in developing psoriatic lesions," Archives of Dermatological Research, vol. 292, no. 12, pp. 590-597, 2000.

[23] G. Salinas, L. Valderrama, G. Palma, G. Montes, and N. G. Saravia, "Detection of amastigotes in cutaneous and mucocutaneous leishmaniasis using the immunoperoxidase method, using polyclonal antibody: sensibility and specificity compared with conventional methods of diagnosis," Memórias do Instituto Oswaldo Cruz, vol. 84, no. 1, pp. 53-60, 1989.

[24] L. C. Reis, E. M. Ramos-Sanchez, and H. Goto, "The interactions and essential effects of intrinsic insulin-like growth factor-i on Leishmania (Leishmania) major growth within macrophages," Parasite Immunology, vol. 35, no. 7-8, pp. 239-244, 2013.

[25] L. P. Carvalho, S. Passos, O. Bacellar et al., "Differential immune regulation of activated $\mathrm{T}$ cells between cutaneous and mucosal leishmaniasis as a model for pathogenesis," Parasite Immunology, vol. 29, no. 5, pp. 251-258, 2007.
[26] O. Bacellar, H. Lessa, A. Schriefer et al., "Up-regulation of Th1type responses in mucosal leishmaniasis patients," Infection and Immunity, vol. 70, no. 12, pp. 6734-6740, 2002.

[27] S. Sukhanov, Y. Higashi, S. Y. Shai et al., "IGF-1 reduces inflammatory responses, suppresses oxidative stress, and decreases atherosclerosis progression in ApoE-deficient mice," Arteriosclerosis, Thrombosis, and Vascular Biology, vol. 27, no. 12, pp. 2684-2690, 2007.

[28] W. J. Lee, "IGF-I exerts an anti-inflammatory effect on skeletal muscle cells through down-regulation of TLR4 signaling," Immune Network, vol. 11, no. 4, pp. 223-226, 2011.

[29] G. Kratz, M. Lake, and M. Gidlund, "Insulin like growth factor-1 and -2 and their role in the re-epithelialisation of wounds; interactions with insulin like growth factor binding protein type 1," Scandinavian Journal of Plastic and Reconstructive Surgery and Hand Surgery, vol. 28, no. 2, pp. 107112, 1994.

[30] D. L. Brown, C. D. Kane, S. D. Chernausek, and D. G. Greenhalgh, "Differential expression and localization of insulin-like growth factors I and II in cutaneous wounds of diabetic and nondiabetic mice," The American Journal of Pathology, vol. 151, no. 3, pp. 715-724, 1997.

[31] R. Blakytny, E. B. Jude, J. Martin Gibson, A. J. Boulton, and M. W. Ferguson, "Lack of insulin-like growth factor 1 (IGF1) in the basal keratinocyte layer of diabetic skin and diabetic foot ulcers," The Journal of Pathology, vol. 190, no. 5, pp. 589-594, 2000.

[32] F. Gong, F. Zhao, S. L. Cheng et al., "Effect of insulin-like growth factor-1 on promoting healing of skin ulcers in diabetic rats," Journal of Biological Regulators and Homeostatic Agents, vol. 33, no. 3, pp. 687-694, 2019.

[33] A. C. Maretti-Mira, M. P. de Oliveira-Neto, A. M. Da-Cruz, M. P. de Oliveira, N. Craft, and C. Pirmez, "Therapeutic failure in American cutaneous leishmaniasis is associated with gelatinase activity and cytokine expression," Clinical and Experimental Immunology, vol. 163, no. 2, pp. 207-214, 2011.

[34] A. L. Bittencourt and A. Barral, "Evaluation of the histopathological classifications of American cutaneous and mucocutaneous leishmaniasis," Memórias do Instituto Oswaldo Cruz, vol. 86, no. 1, pp. 51-56, 1991.

[35] S. Arkins, N. Rebeiz, D. L. Brunke-Reese, A. Biragyn, and K. W. Kelley, "Interferon-gamma inhibits macrophage insulin-like growth factor-I synthesis at the transcriptional level," Molecular Endocrinology, vol. 9, no. 3, pp. 350-360, 1995.

[36] F. Conceição-Silva, J. Leite-Silva, and F. N. Morgado, "The binomial parasite-host immunity in the healing process and in reactivation of human tegumentary leishmaniasis," Frontiers in Microbiology, vol. 9, p. 1308, 2018.

[37] P. Esterre, J. P. Dedet, S. Guerret, M. Chevallier, C. Frenay, and J. A. Grimaud, "Matrix remodelling and fibroblast phenotype in early lesions of human cutaneous leishmaniasis," Pathology, Research and Practice, vol. 187, no. 8, pp. 924-930, 1991. 